\title{
Fatty acid synthase inhibitor orlistat impairs cell growth and down-regulates PD-L1 expression of a human T-cell leukemia line
}

Giorgia Cioccoloni, Angelo Aquino, Maria Notarnicola, Maria Gabriella Caruso, Enzo Bonmassar, Manuela Zonfrillo, Simona Caporali, Isabella Faraoni, Cristina Villivà, Maria Pia Fuggetta \& Ornella Franzese

To cite this article: Giorgia Cioccoloni, Angelo Aquino, Maria Notarnicola, Maria Gabriella Caruso, Enzo Bonmassar, Manuela Zonfrillo, Simona Caporali, Isabella Faraoni, Cristina Villivà, Maria Pia Fuggetta \& Ornella Franzese (2019): Fatty acid synthase inhibitor orlistat impairs cell growth and down-regulates PD-L1 expression of a human T-cell leukemia line, Journal of Chemotherapy, DOI: 10.1080/1120009X.2019.1694761

To link to this article: https://doi.org/10.1080/1120009X.2019.1694761

View supplementary material $₫$

Published online: 28 Nov 2019.

Submit your article to this journal

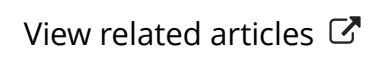

View Crossmark data $\asymp$ 


\title{
Fatty acid synthase inhibitor orlistat impairs cell growth and down-regulates PD-L1 expression of a human T-cell leukemia line
}

\author{
Giorgia Cioccoloni ${ }^{1}$, Angelo Aquino ${ }^{1}$, Maria Notarnicola ${ }^{2 *}$, Maria Gabriella \\ Caruso $^{2 *}$, Enzo Bonmassar ${ }^{1,3}$, Manuela Zonfrillo ${ }^{3}{ }^{\infty}$, Simona Caporali ${ }^{4}$, \\ Isabella Faraoni $^{1}$, Cristina Villivà ${ }^{3}$, Maria Pia Fuggetta ${ }^{3}$, Ornella Franzese ${ }^{1}$
}

${ }^{1}$ Department of Systems Medicine, University of Rome Tor Vergata, Rome, Italy; ${ }^{2}$ Laboratory of Nutritional Biochemistry, National Institute for Digestive Diseases S. de Bellis, Bari, Italy; ${ }^{3}$ Institute of Translational Pharmacology, National Council of Research, Rome, Italy; ${ }^{4}$ Laboratory of Molecular Oncology, IDI-IRCCS Rome, Rome, Italy

\begin{abstract}
Fatty Acid Synthase (FASN) is responsible for the de novo synthesis of fatty acids, which are involved in the preservation of biological membrane structure, energy storage and assembly of factors involved in signal transduction. FASN plays a critical role in supporting tumor cell growth, thus representing a potential target for anti-cancer therapies. Moreover, this enzyme has been recently associated with increased PD-L1 expression, suggesting a role for fatty acids in the impairment of the immune response in the tumor microenvironment. Orlistat, a tetrahydrolipstatin used for the treatment of obesity, has been reported to reduce FASN activity, while inducing a sensible reduction of the growth potential in different cancer models. We have analyzed the effect of orlistat on different features involved in the tumor cell biology of the T-ALL Jurkat cell line. In particular, we have observed that orlistat inhibits Jurkat cell growth and induces a perturbation of cell cycle along with a decline of FASN activity and protein levels. Moreover, the drug produces a remarkable impairment of PD-L1 expression. These findings suggest that orlistat interferes with different mechanisms involved in the control of tumor cell growth and can potentially contribute to decrease the tumor-associated immunepathogenesis.
\end{abstract}

KEYWORDS: FASN; leukemia; PD-L1

\section{Introduction}

Fatty Acid Synthase (FASN) is a multifunctional homodimeric enzyme responsible for de novo synthesis of fatty acids. ${ }^{1,2}$ FASN is composed of six different enzymatic domains working in a cyclical loop to generate palmitate starting from the precursor metabolites acetyl-CoA and malonyl-CoA. Palmitate induces profound changes in the biophysical nature of cancer cell membrane, promoting a more efficient assembly of the molecules involved in cell signaling pathways and required for sustained tumor cell proliferation and survival. ${ }^{3}$

Correspondence to: Fuggetta Maria Pia, Institute of Translational Pharmacology, National Council of Research, Rome, Italy. Email: mariapia.fuggetta@ift.cnr.it

G.C. and A.A. contributed equally to this work.

* Laboratory of Nutritional Biochemistry, National Institute of Gastroenterology-Research Hospital, "S. de Bellis", Castellana Grotte, Bari, Italy.

(4) Supplemental data for this article can be accessed here: https:// doi.org/10.1080/1120009X.2019.1694761.
Furthermore, the more regular architecture of cell membrane promoted by FASN inhibition leads to a higher sensitivity of tumors to cytotoxic chemotherapies. ${ }^{4,5}$ Differently, endogenous de novo fattyacid synthesis due to FASN activity is barely effective in normal tissues, because of the substantial exogenous intake of fatty acids from the diet. ${ }^{6,7}$ Fatty acid accumulation correlates with a dysregulated expression of FASN while playing a more general regulatory role on gene expression. ${ }^{5,8} \mathrm{~A}$ direct correlation with tumor stage has been also described, suggesting that FASN not only provides a metabolic advantage favoring cancer cell survival and proliferation but also supports a particularly aggressive tumor phenotype. ${ }^{9-12}$ Expression of FASN in cancer cells is controlled by several growth factors and receptors including the epidermal growth factor (EGFR) and the estrogen receptor $(\mathrm{ER})^{8}$ and signaling pathways including 
phosphatidylinositol-3-kinase (PI3K)-AKT and mitogen-activated protein kinase (MAPK). ${ }^{13}$ These observations indicate FASN as a potential target for anti-cancer therapies. ${ }^{14}$

Several studies show that orlistat, a tetrahydrolipstatin inhibitor of gastric lipases used for the treatment of obesity, determines the inhibition of FASN, along with suppression of angiogenesis and inhibition of growth in different cancer cell line models. ${ }^{15-18}$ Interestingly, this compound has also been shown to reduce protein levels of O6-methylguanine-DNA methyltransferase (MGMT), a DNA repair enzyme playing an essential role in the protection against DNA damage provoked by DNA methylating compounds. ${ }^{19}$ Orlistat generates a covalent adduct with the FASN thioesterase domain, ${ }^{20}$ thus inhibiting irreversibly the enzymatic activity. This is followed by the activation of apoptotic cascade, mediated by caspase- 8 , negative regulation of the mTOR pathway ${ }^{21}$ and cell cycle arrest at the G1/S phase. ${ }^{22}$ Moreover, FASN is involved in the development of resistance to NK cell cytotoxicity of cisplatin-resistant cancer cells, ${ }^{23}$ and its inhibition by orlistat increases breast cancer cell susceptibility to anticancer therapies. ${ }^{24}$

Programmed Cell Death- 1/Programmed Cell Death-Ligand 1 (PD-1/PD-L1) pathway is a wellknown immune checkpoint. ${ }^{25,26}$ Increased PD-L1 expression in tumor cells is associated with the development of resistance to T-cell-mediated cytotoxicity, ${ }^{27,28}$ and clinical approaches targeting PD-1/PDL1 engagement substantially improve T-cell-mediated anti-tumor immune function. ${ }^{29}$ Inhibition of FASN has been recently associated with reduced levels of PD-L1, through the impairment of a FASN-TGF $\beta 1$ PD-L1 axis, ${ }^{23}$ suggesting that fatty acid accumulation might also contribute to impair the immune response in the tumor microenvironment. ${ }^{30,31}$

Most studies have been performed on solid tumors, and only few reports describe the effect of orlistat in hematological malignancies. In chronic lymphocytic leukemia, orlistat induces dose-dependent apoptosis, but the mechanism underlying this effect has not been completely elucidated yet. ${ }^{32,33}$

T-cell acute lymphoblastic leukemia (T-ALL) is characterized by several genetic alterations, causing dysregulation in cell growth, differentiation and survival. Although the prognosis has improved during last decades, the clinical outcome of patients affected by primary resistant leukemia remains poor. ${ }^{34,35}$

Orlistat shows promising antineoplastic activity, either alone or in combination with other cytotoxic compounds, ${ }^{5}$ or with drugs targeting glycolysis and glutaminolysis. ${ }^{36}$ In the present work we have analyzed the effect of orlistat on selected key features of tumor cell biology of the T-ALL Jurkat cell line.
Taking into account the role of PD-1/PD-L1 immune checkpoint in anti-tumor T-cell function, we have also investigated the effect of orlistat on the expression of PD-L1.

\section{Materials and methods Cell line and drug treatment}

The human $\mathrm{CD}^{+} \mathrm{T}$ cell leukemia Jurkat cell line was obtained from the American Type Culture Collection (ATCC, Rockville, MD, USA). The human promyelocytic HL-60 cells were generously provided by Dr Stefania D'Atri (Laboratory of Molecular Oncology, IDI-IRCSS, Rome, Italy). Cells were cultured at $37^{\circ} \mathrm{C}$ in $5 \% \mathrm{CO}_{2}$ humidified atmosphere in RPMI-1640 (Sigma-Aldrich, Saint Louis, Missouri, USA) containing 10\% heat-inactivated FCS (Sigma-Aldrich), $2 \mathrm{mM}$ L-glutamine (Sigma-Aldrich), and $50 \mu \mathrm{g} / \mathrm{ml}$ gentamicin (Euroclone, Milan Italy), hereafter referred to as Complete Medium (CM). Orlistat (Sigma-Aldrich) was dissolved in sterile DMSO (Sigma-Aldrich) at $20 \mathrm{mM}$ concentration, then aliquoted and stored at $-20^{\circ} \mathrm{C}$. Hydrosoluble Dexamethasone Cyclodextrin complex (hereafter designed as DEX) was obtained from Sigma-Aldrich, and Methotrexate (MTX), sodium salt was obtained from Pfizer Inc. Combination experiments with orlistat were carried out with $10 \mu \mathrm{M}$ DEX and graded concentrations of MTX and evaluated after $72 \mathrm{~h}$ of culture. In particular Jurkat cells were exposed to suboptimal concentrations of orlistat or graded amounts of MTX ranging from 0.0125 and $0.1 \mu \mathrm{M}$ alone or to both drugs combined at a fixed ratio (i.e orlistat: MTX, 200:1).

\section{Fatty acid solutions}

Fatty acid-free bovine serum albumin (BSA) and Sodium Palmitate (SP) were purchased from Sigma-Aldrich. SP was prepared in a $1800 \mathrm{mM}$ stock solution in $90 \%$ ethanol as described by Sadowsky et al. ${ }^{37}$ Briefly, the solution was incubated at $70^{\circ} \mathrm{C}$ for $30 \mathrm{~min}$ by repeatedly vortexing, diluted to $20 \mathrm{mM}$ in a $5.5 \%$ BSA solution in PBS without fatty acids and then stored at $-20^{\circ} \mathrm{C}$ until use at the final concentration of $5 \mu \mathrm{M}$, as described. ${ }^{37}$ A 5.5\% BSA solution in fatty acid-free PBS was used as control.

\section{Cell growth analysis}

Jurkat cells were suspended in $25 \mathrm{~cm}^{2}$ flask (Corning, New York, USA) and cultured for 24 hours at $37^{\circ} \mathrm{C}$ in a $5 \% \mathrm{CO}_{2}$ before incubation with orlistat at the concentrations and the times indicated. Total number of viable cells was counted manually and percent cell 
viability was estimated according to Trypan blue exclusion assay.

\section{MTT assay}

Cytotoxicity of orlistat was assessed through MTT assay. Jurkat cells $\left(10^{5}\right.$ cells/well $)$ were seeded in 96-wells microtiter dishes (BD Falcon ${ }^{\mathrm{TM}}$, Franklin Lakes, New Jersey, USA) in $100 \mu \mathrm{l}$ of $\mathrm{CM}$ and treated for 72 hours at $37^{\circ} \mathrm{C}$ and $5 \% \mathrm{CO}_{2}$ with different concentrations of orlistat as indicated.

Viability was determined by incubating cells with $20 \mu 1$ MTT dye $\left(5 \mathrm{mg} / \mathrm{ml}\right.$ in PBS) at $37^{\circ} \mathrm{C}$ for $3 \mathrm{~h}$. Cells were incubated with $100 \mu$ lysis buffer $(20 \%$ SDS in $50 \%$ dimethylformamide) at $37^{\circ} \mathrm{C}$ for $1 \mathrm{~h}$. The extent of formazan production was determined by optical density (OD) detection at $570 \mathrm{~nm}$ by Multiskan EX (Labsystems, Milan, Italy) analyzer.

\section{FACS analysis of cell cycle, apoptosis, PD-L1 and intracellular FASN expression}

Cells were suspended into $25 \mathrm{~cm}^{2}$ flask (Corning) and allowed to grow for 24 hours at $37{ }^{\circ} \mathrm{C}$ in a $5 \%$ $\mathrm{CO}_{2}$ atmosphere followed by incubation with different concentrations of orlistat, ranging from 20 to $60 \mu \mathrm{M}$ for 72 hours, then fixed overnight with $70 \%$ ethanol at $4{ }^{\circ} \mathrm{C}$, and incubated with $10 \mu \mathrm{g} / \mathrm{ml}$ of RNase A and $50 \mu \mathrm{g} / \mathrm{ml}$ of propidium iodide (PI) for 1 hour at room temperature in the dark. Samples were acquired on FacsCalibur (BD Becton Dickinson, Franklin Lakes, New Jersey, USA). The acquired FACS data were analyzed by ModFit LT software (Verity Software House, Inc., Topsham, Maine, USA) to quantify the fraction of cells in each phase of the cycle, i.e. the percentage of cells detected in sub G1, G1, S and G2/M phases. Cell surface-staining of PD-L1 and PD-1 was performed by incubating cells with PE-antiPD-L1 or anti-PD-1 (BD Pharmigen) antibody at $4{ }^{\circ} \mathrm{C}$ for $30 \mathrm{~min}$. Analysis of FASN expression was performed by intracellular flow cytometry detection, through cell permeabilization with Cytofix/ Cytoperm (BD Pharmingen) and staining with anti FASN monoclonal antibody (mAb) (Thermo Fisher Scientific, Waltham, Massachusetts, USA) at $4{ }^{\circ} \mathrm{C}$ for $30 \mathrm{~min}$ and subsequent incubation with secondary anti IgG FITC-labeled $\mathrm{Ab}$, by Dako (Dako Glostrup Denmark).

\section{Caspase-3/7 activity}

Caspase-3/7 activity was assayed using the Caspase-Glo 3/7 Assay (Promega, Madison, Wisconsin, USA). Briefly, $15 \times 10^{5}$ cells/well were treated for 72 hours at $37^{\circ} \mathrm{C}$ in a $5 \% \mathrm{CO}_{2}$ with different concentrations of orlistat $(20 \mu \mathrm{M}$ and $40 \mu \mathrm{M})$ on a 96 well plate (BD Falcon ${ }^{\mathrm{TM}}$, BD Becton Dickinson, Franklin Lakes, New Jersey, USA).
Then, cells were incubated in a cell lysis solution containing a luciferase substrate derivative specifically cleaved by active caspase-3/7, resulting in the conversion of the substrate into a luminescent signal [RLU, Ultra- Glo ${ }^{\mathrm{TM}}$ Recombinant Luciferase (Promega)] at room temperature for 1 hour. The luminescent signal was then normalized to cell number.

\section{Western blot}

Cells were isolated at times indicated and extracts obtained by lysing cells in a $\left(12.5 \mathrm{mM} \mathrm{Na} \mathrm{HPO}_{4}, \mathrm{pH}\right.$ 7.2; $94 \mathrm{mM} \mathrm{NaCl} ; 50 \mathrm{mM} \mathrm{NaF} ; 1 \%$ Triton X-100; $2 \mathrm{mM}$ EGTA) extraction buffer, additioned with $1 \%$ protease inhibitor cocktail (Sigma-Aldrich), 0.5\% saponin (Sigma-Aldrich) and $1 \mathrm{mM} \mathrm{Na} \mathrm{NO}_{4}$ (SigmaAldrich). Protein concentration was evaluated using Bio-Rad Protein Assay Dye and samples stored at $-80^{\circ} \mathrm{C}$. Proteins were separated on $10 \%$ polyacrylamide pre-cast gels (NuPAGE® Novex Bis-Tris, Invitrogen, Life Technologies, Grand Island, NY, USA), using XCell SureLock ${ }^{\mathrm{TM}}$ Mini-Cell apparatus (Invitrogen) following the manufacturer's instructions. After electrophoretic separation, proteins were transferred to Hybond-ECL nitrocellulose filters (GE Healthcare Life Sciences, Pittsburg, PA, USA), probed with the specific primary Abs against FASN (Thermo Fisher Scientific, (Waltham, Massachusetts, USA) and $\beta$ actin (rabbit, Sigma-Aldrich), then exposed to the corresponding secondary alkaline phosphate-conjugated $\mathrm{Ab}$ and protein bands were detected with the WesternBreeze chemiluminescent immunodetection kit (Invitrogen). The quantification of all band intensities was performed with GS-710 Calibrated Imaging Densitometer and analyzed with Quantity One software, version 4.1.1 (Bio-Rad Laboratories). FASN expression was evaluated on the basis FASN: $\beta$ actin ratio.

\section{FASN activity assay}

Cells were suspended in lysis buffer ( $25 \mathrm{mM}$ TRIS$\mathrm{HCl}(\mathrm{pH}=7.4), 1 \mathrm{mM}$ DTT, $1 \mathrm{mM} \mathrm{MgCl} 2$ e $1 \mathrm{mM}$ di PMSF) and centrifuged at $10000 \mathrm{rpm}$ for $10 \mathrm{~min}$. An aliquot of supernatant $(50 \mu \mathrm{l})$ was pre-incubated with $100 \mathrm{mM}$ potassium phosphate buffer, $\mathrm{pH} 7$ at $37^{\circ} \mathrm{C}$ for $15 \mathrm{~min}$. Subsequently, $20 \mu \mathrm{l}$ of reaction mix $(2.5 \mathrm{mM}$ NADPH, $1.25 \mathrm{mM}$ acetyl-CoA, $1.25 \mathrm{mM}$ malonyl-CoA and $0.02 \mathrm{mM} \quad 2-14 \mathrm{C}-$ malonyl-CoA $\quad(52 \mathrm{mCi} / \mathrm{mmol}, \quad$ Amersham Biosciences, UK) were added and samples were incubated at $37^{\circ} \mathrm{C}$ for $10 \mathrm{~min}$. Reactions were stopped by the addition of $500 \mu 11 \mathrm{~N} \mathrm{HCl} /$ methanol $(6: 4, v: v)$; fatty acids were extracted with $1 \mathrm{ml}$ of petroleum ether and incorporation of 2-14Cmalonyl-CoA was analyzed by scintillation counting. FASN activity was expressed as picomoles of 

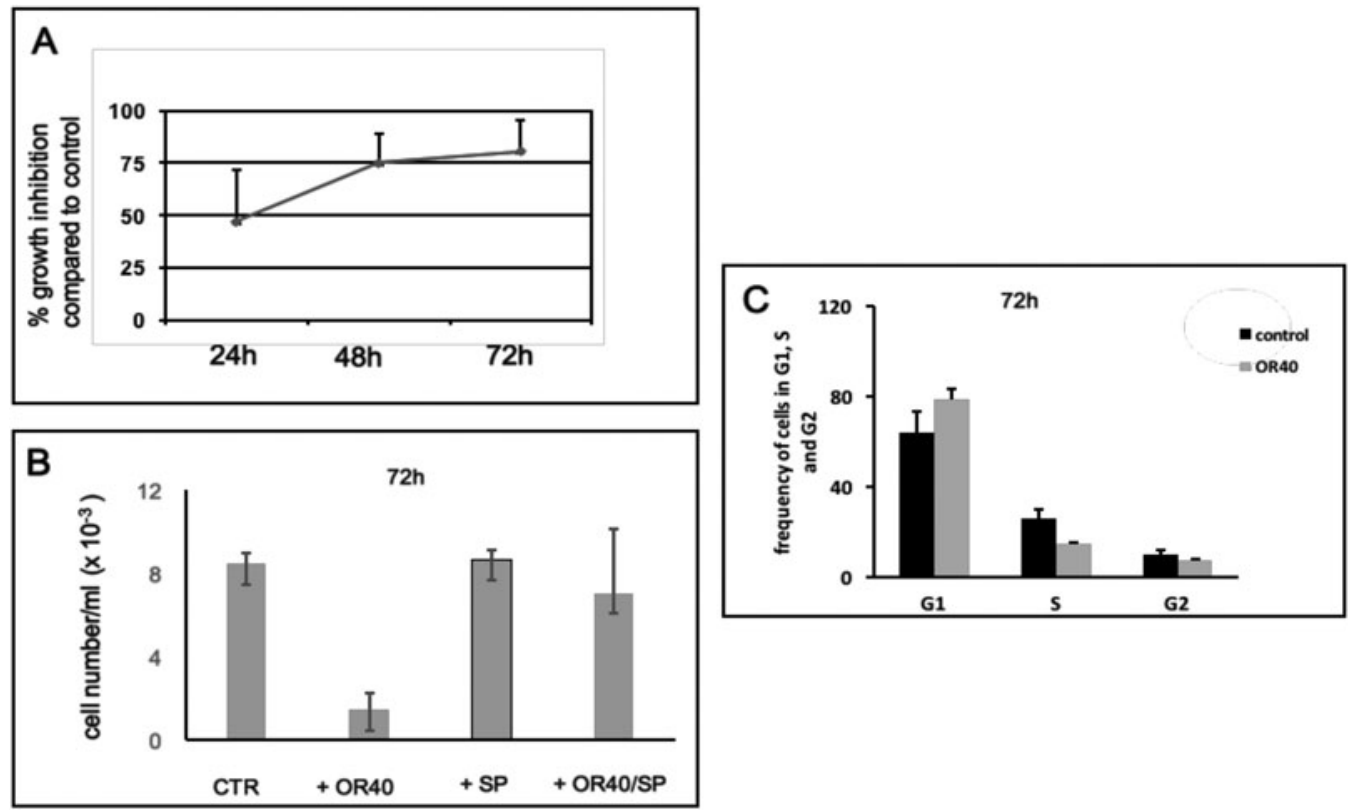

Figure 1. Orlistat inhibits Jurkat cell proliferation and induces cell cycle G1 retardation. Panel A: Time course analysis of the inhibitory effect of $40 \mu \mathrm{M}$ orlistat (OR40) on proliferation of leukemia T-cell line Jurkat. Each value represents the mean of three independent experiments. Panel B: Partial rescue of orlistat-mediated cytotoxicity with exogenous palmitate (SP, used at $5 \mu \mathrm{M}$ concentration). Graph shows the antiproliferative effect of orlistat and the cell growth rescue obtained by OR40/SP co-treatment. SP induces a slight and non significant increase of Jurkat cell growth when administered alone. Each value represents the mean of two independent experiments. Bars indicate SE. Panel C. Orlistat induces cell cycle perturbation in Jurkat cells. Graph shows the frequency of distribution of Jurkat cells in different cell cycle phases after treatment with $40 \mu \mathrm{M}$ orlistat (OR40) and related control of a representative experiment (FACS analysis). Statistical significance was attributed to results with ${ }^{*} p<0.05$ after parametric test (student t-test) or non-parametric test (Wilcoxon-Mann-Whitney).

incorporated 2-14 C-malonyl- CoA per minute per milligram of total proteins ( $\mathrm{pmol} / \mathrm{min} / \mathrm{mg}$ protein).

\section{Statistical analysis}

Paired t test or non-parametric Wilcoxon test were performed to analyze differences between controls and treated cells. All statistical analysis results were considered significant when $p$ values were less than 0.05 with a $95 \%$ confidence interval. When required, mean percentages relative to different experiments were subjected to angular transformation in order to obtain normally distributed values manageable by Student $t$ test.

To study the effects of combined treatment with orlistat and MTX on Jurkat cell proliferation, we used the median effect method described by Chou and Talalay. ${ }^{38}$ According to this method, a parameter called combination index (CI) is calculated based on the following equation:

$$
\begin{aligned}
\mathrm{CI}= & \mathrm{DA} /(\mathrm{Dx}) \mathrm{A}+\mathrm{DB} /(\mathrm{Dx}) \mathrm{B} \\
& +\alpha \mathrm{DADB} /(\mathrm{Dx}) \mathrm{A}(\mathrm{Dx}) \mathrm{B}
\end{aligned}
$$

where DA and DB are the concentrations of drug $\mathrm{A}$ and drug $\mathrm{B}$ that have a " $\mathrm{x}$ "effect when used in combination at a fixed ratio, (Dx)A and (Dx)B are the concentrations of drug A and drug $\mathrm{B}$ that have the same effect when used individually, and $\alpha=1$ or 0 depending on whether the two drugs are assumed to be mutually nonexclusive (i.e they have different modes of action or act independently) or mutually exclusive (i.e. they act on the same targets). According to this method, for each level of effect exerted by the drug combination, synergy is indicated by $\mathrm{CI}<1$, additivity by $\mathrm{CI}=1$, and antagonism by CI $>1$. The CalcuSyn software (Cambridge Biosoft, Cambridge, UK) was used to calculate the CI values from the experimentally determined concentrationresponse curves for orlistat, MTX or the combinations of both drugs. The CI was calculated according to the more stringent statistical assumption that orlistat and MTX are mutually nonexclusive drugs.

\section{Results}

\section{Orlistat inhibits proliferation, reduces viability and induces cycle perturbations, while does not induce apoptosis in Jurkat cells}

Preliminary MTT experiments were carried out with graded concentrations of orlistat, in order to identify the dose able to induce $50 \%$ cell growth inhibition (IC50) on day 3 of culture. IC50 was $32.87 \mu \mathrm{M} \quad$ (confidential limits, 37.83-28.58) (Supplementary Figure 1).

Hereafter, in the present study we decided to employ the $40 \mu \mathrm{M}$ concentration in order to obtain a substantial cytotoxic activity slightly exceeding the upper limit of the IC50 confidential interval. 

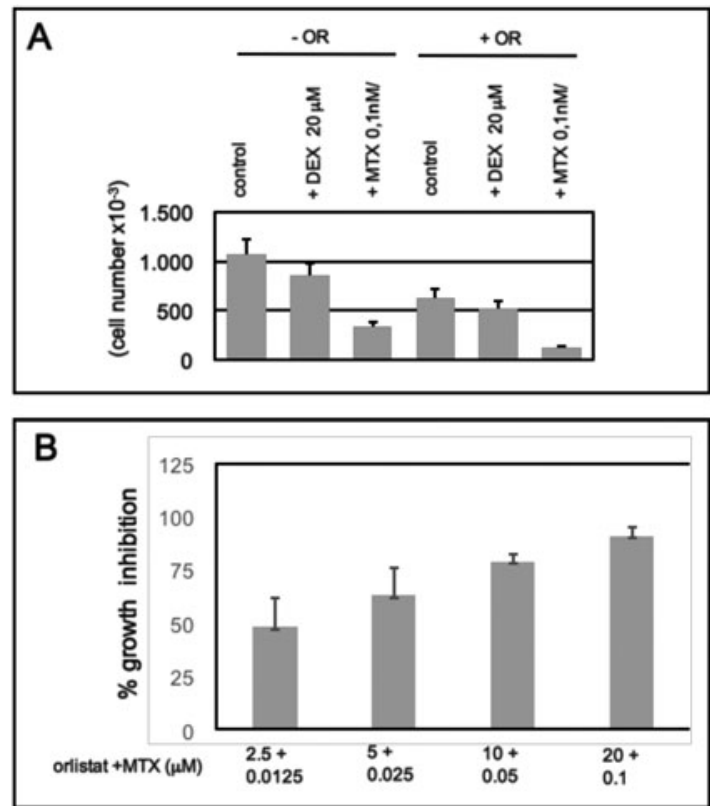

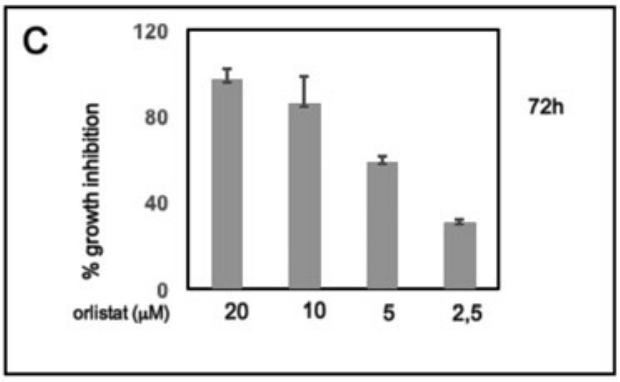

Figure 2. Panel A. The combined use of OR40 with both DEX and MTX is beneficial in terms of Jurkat cell growth inhibition compared with orlistat alone. Analysis of the inhibitory effect of $40 \mu \mathrm{M}$ orlistat (OR40) in combination with DEX or with MTX on growth capacity of leukemia T-cell line Jurkat. Bars indicate SE. Panel B. Effect of combined treatment with orlistat and MTX on Jurkat cell growth. Cells were cultured in the presence of sub-optimal concentrations of orlistat and graded amounts of MTX for $72 \mathrm{~h}$. Orlistat and MTX were combined at the constant ratio of 200:1. Cell growth was evaluated by the MTT assay. Each value represents the mean of four independent experiments, with bars indicating SE of the mean. CI values (see statistical analysis) at a level of cell growth inhibition of $50 \%, 75 \%$ and $90 \%$ were $0.68 \pm 0.09,0.66 \pm 0.11$ and $0.67 \pm 0.16$, respectively. Panel C. Effect of orlistat on acute promyelocytic leukemia cell line HL-60 cell growth. Cells were cultured in the presence of graded concentrations of orlistat. The mean of IC50 was 4.03 (with confidential limits of 5, 7-2.9). Each value represents the mean of two independent experiments, with bars indicating SE. Statistical significance was attributed to results with ${ }^{*} \mathbf{p}<0.05$ after parametric test (student t-test) or non-parametric test (Wilcoxon-Mann-Whitney).

Also, $40 \mu \mathrm{M}$ represents the concentration used in most experiments of our previous work. ${ }^{19}$

Jurkat cell proliferation rate with or without $40 \mu \mathrm{M}$ orlistat (OR40) was analyzed over 3 days of culture by cell count. A sensible growth inhibition was observed already after 24 hours and peaked after 72 hours of treatment (Figure1 A shows the mean percentages of cell growth inhibition values obtained from three out of seven independent experiments performed). Similar results were obtained using MTT assay (data not shown).

Next we investigated whether deprivation of the palmitate presumably produced by orlistat-mediated FASN inhibition could play a role in the cytotoxic/cytostatic effects of the drug. Rescue experiments were performed by supplementing orlistat-treated Jurkat cells with exogenous palmitate (SP). Addition of palmitate did not substantially increase the growth of control Jurkat cells, while partly and significantly reverted the pronounced growth inhibition induced by orlistat (Figure 1B, where histograms represent the mean values obtained from two independent experiments). These results indicate that the anti-proliferative effect of orlistat was, at least in part, the result of end product deprivation due to FASN inhibition.

We then investigated whether orlistat-mediated inhibition of Jurkat cell growth was associated with cell cycle perturbation. FACS analysis performed on Jurkat cells after $72 \mathrm{~h}$ treatment showed an increase in the extent of cell cycle arrest at G1 phase $(\Delta \%=22,68 \%)$ compared to control cells and a reduction of the frequency of cells in $\mathrm{S}$ and $\mathrm{G} 2$ phases $(\Delta \%=-44,80 \%$ and $\Delta \%=-28,41 \%$ respectively, Figure 1C).

Furthermore, after $72 \mathrm{~h}$ of orlistat treatment, only a small increase in the activity of caspase-3/7 was observed in treated cells compared to controls $(\Delta \%=13,22 \%)$, in association with a slight rise in the percentage of apoptotic cells after treatment at $24 \mathrm{~h} \quad(\Delta \%=3.70 \%), \quad 48 \mathrm{~h} \quad(\Delta \%=3.42)$ and $72 \mathrm{~h}$ $(\Delta \%=4.8)$, analyzed by sub-G1 DNA peak. These data suggest that orlistat-mediated inhibition of Jurkat cell growth is only marginally attributable to the induction of apoptosis.

Moreover, since several newly-developed targeting drugs are used in combination with classic chemotherapeutic agents, we designed experiments aimed at analyzing the combined effects of orlistat with dexamethasone (DEX) and methotrexate (MTX), commonly used drugs for the management of lymphoblastic leukemia. The combined use of OR40 with both DEX and MTX proved beneficial in terms of Jurkat cell growth inhibition compared with orlistat alone (Figure 2A). 

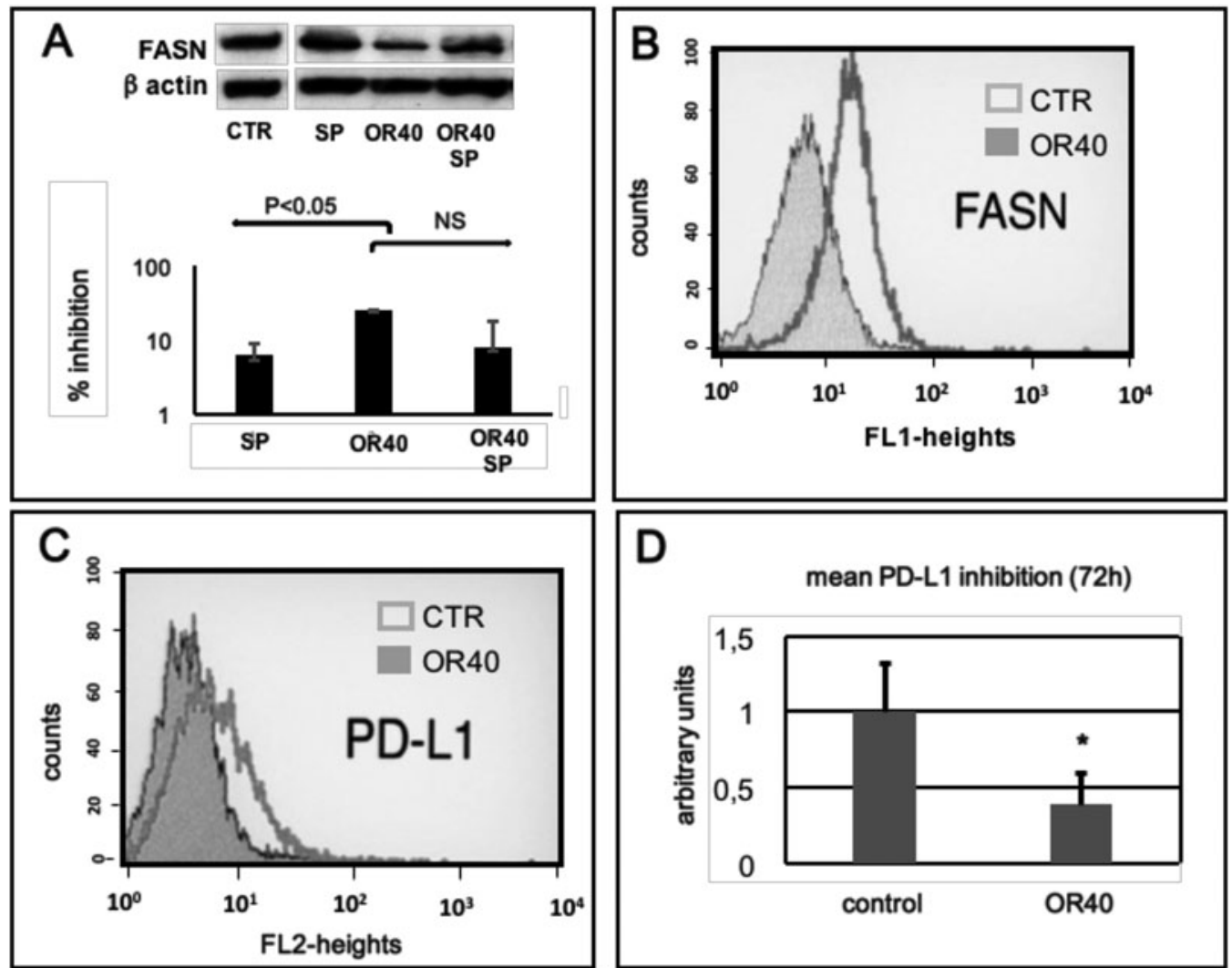

Figure 3. Orlistat reduces FASN and PD-L1 expression. Panel A: Representative experiment showing the effect of $40 \mu \mathrm{M}$ orlistat treatment (OR40) on FASN expression tested on whole cell extracts of Jurkat cells after $72 \mathrm{~h}$ treatment (Western Blot). Gel loading control and quantification was performed analyzing $\beta$ actin expression. Histogram graph shows the means of percent inhibition of FASN expression produced by treatment of Jurkat cells with SP $(5 \mu \mathrm{M})$, OR $(40 \mu \mathrm{M})$ or OR + SP from 3 independent experiments. In order to utilize percent inhibition values for parametric statistics, the percentages were subjected to angular transformation to generate normally-distributed data according to Bonmassar et al. ${ }^{39}$ ( $p<0.05$ according paired $t$ test). Bars indicate SE. NS, not significant. Panel B: Representative FACS histogram plot showing the effect of $40 \mu \mathrm{M}$ orlistat treatment (OR40) on intracellular FASN expression of Jurkat cells. Panel C: Representative FACS histogram plot showing the effect of $40 \mu \mathrm{M}$ orlistat treatment (OR40) on PD-L1 expression of Jurkat cells. Panel D: Histogram graph showing the mean percentage inhibition (arbitrary units) mediated by $40 \mu \mathrm{M}$ orlistat treatment (OR40) on PD-L1 expression of Jurkat cells and the relative SE obtained from 3 independent experiments. The mean percentage inhibition and the relative SE were calculated after angular transformation of each data $(p<0.05$ according paired $t$ test).

This approach can potentially provide therapeutic anti-cancer benefit by reducing tumour growth potential, while reducing drug resistance and allowing drug dose reduction. Therefore, we further extended our studies on the combined use of orlistat and MTX and additional experiments were carried out in order to ascertain whether this combination could result in a synergistic inhibitory effect. Jurkat cells were incubated for $72 \mathrm{~h}$ with suboptimal concentrations of orlistat $(2.5,5,10$, $20 \mu \mathrm{M})$ and graded concentrations of MTX $(0.0125,0.025,0.05,0.1 \mu \mathrm{M})$, individually (data not shown) and in combination to obtain a constant orlistat to MTX ratio of 200:1. The CI at level of growth inhibition of $50 \%, 75 \%$ and $95 \%$ was calculated according to Chou and Talalay. ${ }^{38}$ The results showed that at the concentrations tested, the effects of orlistat and MTX were moderately synergic (see legend of Figure 2B).

Interestingly, additional experiments carried out on the HL-60 cell line, an acute promyelocytic leukemia, showed a higher cytotoxic effect of orlistat, with an average IC50 of 4.03 (with confidential limits of 5, 7-2.9, see Figure 2C). This suggests that orlistat can represent a valid option for the treatment of different hematological malignancies.

\section{Effect of orlistat alone, or in combination with exogenous palmitate on FASN expression}

Inhibition of FASN is considered one of the main mechanisms underlying the antitumor activity of orlistat. We analyzed whether the inhibition of Jurkat cell growth was related to a down-regulation of FASN expression and a decline of FASN protein was observed after 48 (not shown) and $72 \mathrm{~h}$. The results of immunoblot analysis of a representative experiment (Figure 3A) show that treatment with OR40 for $72 \mathrm{~h}$ is followed by a decrease of FASN expression compared with untreated cells. According with densitometric analysis, performed on 3 experiments, OR40 in combination with 
palmitate showed a trend toward reduction of the OR effect on FASN expression, although it did not reach statistical significance. These results support a critical role of FASN in Jurkat cell survival and duplication. The OR40-mediated decrease of FASN expression was confirmed by intracellular cytofluorimetric analysis which revealed a reduced expression of the enzyme in Jurkat cells treated with OR40 for $72 \mathrm{~h}$, as compared with controls, either in terms of percentage of FASN expressing cells (not shown) or Mean Fluorescence Intensity (see Materials and Methods Section), (Figure 3B). Noteworthy, this reduction of FASN expression was accompanied by a specific and concentration dependent inhibition of enzymatic activity $(2.0 \pm 0.2 \mathrm{pmol} / \mathrm{min} / \mathrm{mg}$ protein detected after treatment with $40 \mu \mathrm{M}$ orlistat compared to $10.9 \pm 0.7 \mathrm{pmol} / \mathrm{min} / \mathrm{mg}$ protein observed in control cells in a representative experiment).

\section{Orlistat down-regulates PD-L1 expression in Jurkat cells}

PD-L1 is widely expressed in several cancer types and the engagement of the PD-1/PD-L1 pathway suppresses effector $\mathrm{T}$-cell generation and critical functional parameters, including cytokine production and anti-tumor cytotoxicity. ${ }^{25,26}$ Inhibition of FASN has been associated with reduced levels of PD-L1 in cisplatin-resistant lung cancer cells. ${ }^{23}$ In our model, treatment of Jurkat cells with $40 \mu \mathrm{M}$ orlistat induced a marked reduction of PD-L1 expression, as showed by the representative histogram plot (Figure 3C) and by results obtained in 3 different experiments and expressed in terms of mean percentage inhibition in Figure 3D.

Since Jurkat cell line could serve dual actors for experimental models, either as leukemic tumor cells or immune effector CD4+ T lymphocytes, we analyzed whether also PD-1 expression was modified upon orlistat treatment. Preliminary experiments showed that treatment of Jurkat cells with $40 \mu \mathrm{M}$ orlistat was able to induce a strong reduction of PD-1 expression (MFI 563 of treated cells versus 1206 of controls) detected in correspondence of the same $72 \mathrm{~h}$ time interval considered for the assessment of PD-L1.

\section{Discussion}

Cancer tissues frequently overexpress FASN, as demonstrated in a broad range of different types of tumors including breast, prostate, colorectal, bladder, ovary, lung, osteosarcoma and non-Hodgkin lymphomas. ${ }^{14}$ Differently, normal, non-tumor tissues adjacent to the tumor, rarely show FASN expression. ${ }^{6,7}$ A great number of preclinical studies have determined that fatty acids and de novo lipogenesis (DNL) are crucial players in the survival and proliferation of different types of tumors. FASN plays critical roles in different aspects of the tumor biology involved in cell metabolism but also integrating signaling pathways, contributing to post-translational palmitoylation and impacting membrane structure and function. ${ }^{40}$ Cancer cell metabolism is characterized by high levels of glycolysis, glutaminolysis and de novo synthesis of fatty acids, resulting from the activation of cancerpromoting factors including c-Myc and NF- $\kappa \mathrm{B}$ and the inactivation of tumor suppressor genes such as p53 and PTEN. All these factors converge at the level of PI3K/AKT and the ERK/MAPK pathways, which promote the generation of the energy required for sustained tumor cell growth and proliferation. Interestingly, the PI3K/AKT and ERK/ MAPK pathways exert many of their metabolicrelated activities upon activation of the mTOR Complex-1 (mTORC1), which drives ATP-consuming cellular processes necessary for cell growth and proliferation. ${ }^{41} \mathrm{Up}$ to date, TVB-2640, the first FASN inhibitor, has entered into clinical trials. ${ }^{42,43}$ Based on the observation that FASN is expressed at a much higher extent in tumor cells, as a prerequisite for tumor cell growth and survival, compared to normal counterparts, the enzyme can be considered a typical tumor-associated drug target.

Although the anticancer properties of orlistat are supported by a large number of in vitro and in vivo studies, the use of this drug has some limitations. In particular, a main challenge in the employment of orlistat as a chemotherapeutic is its poor bioavailability. ${ }^{44}$ To overcome these challenges the employment of orlistat-loaded micellar NPs (NP-orlistat) and orlistat-loaded folate receptor-targeted micellar NPs (Fol-NP-orlistat) ${ }^{45}$ has recently been reported. These formulations increase the solubility, stability, and efficacy of the drug and allow to more selectively target cancer cells while reducing the potential toxic effects on normal tissues.

Noteworthy, we report here that orlistat exerts a strong anti-proliferative effect and interferes with the cell cycle of the T-LLA Jurkat cell line. The anti-proliferative effect of orlistat appears to be associated with FASN inhibition as it was partially reverted by co-treatment of Jurkat cells with SP, the final product of FASN activity. This aspect is of clinical relevance as recently shown by Czumaj et al. ${ }^{46}$ since SP can be present at high levels in the serum of colon cancer patients, leading to the requirement of higher doses of orlistat for possible therapeutic success. We found also that, beside the inhibition of the enzymatic activity, orlistat is able 
to reduce FASN protein levels. Interestingly, FASN inhibitors induce accumulation of ubiquinated FASN, ${ }^{47}$ so one hypothesis is that orlistat may be able to bind and inactivate FASN while promoting a proteasome-dependent degradation process.

Another interesting issue worthy of consideration is the role of fatty acids in the tumor microenvironment and how they may affect anti-tumor responses. Increased PD-L1 expression in tumor cells has been associated with the development of resistance to NK cell-mediated cytotoxicity. ${ }^{23} \mathrm{PD}$ 1/PD-L1 pathway is a well-known immune checkpoint $^{25,26}$ and clinical applications aimed at its blockade significantly enhance anti-tumor $\mathrm{T}$ cellmediated immune function. ${ }^{27-30} \mathrm{We}$ observed that Jurkat cells exposed to orlistat show a substantial reduction of PD-L1 expression. This finding points out a potential critical relevance for this compound in antagonizing the immunosuppressive landscape at the tumor site, differently from what observed with several chemotherapeutic agents [e.g. 5-fluorouracil, carboplatin, doxorubicin gemcitabine, paclitaxel] which induce a dose-dependent increase of PD-L1 in human and mouse cancer models. ${ }^{48-51}$

Clinical studies indicate that increased tumor cell expression of PD-L1 correlates with poor clinical outcomes in hematological diseases and in solid tumors as well. ${ }^{52-54}$ Similar to what observed in other malignancies, PD-L1 expression has been inconstantly detected on human AML cells. ${ }^{55}$ Beside its critical role in maintaining an immunosuppressive tumor microenvironment by impairing T-cell mediated anti-tumor responses, ${ }^{56}$ PD-L1 mediates also critical cell-intrinsic signals which regulate immune-independent tumor growth and metabolism, ${ }^{57}$ while mediating resistance to chemotherapeutic agents. ${ }^{58}$ Therefore, the decrease of PD-L1 expression mediated by orlistat can potentially reduce the tumor immune-pathogenesis while contributing to the inhibitory effect on tumor cell growth potential and metabolism.

TGF- $\beta 1$ has been found to be a FASN downstream signaling molecule modulating the PD-L1 levels in cisplatin-resistant cells. ${ }^{23}$ Accordingly, a critical role for the FASN-TGF $\beta 1$ axis can be suggested to explain orlistat-mediated PD-L1 downregulation. Moreover, PD-L1 expression is tightly regulated by the AKT pathway both in vitro and in vivo, ${ }^{59}$ while FASN inactivation is associated with impairment of this kinase. ${ }^{60}$ Therefore, the orlistat-mediated decline of PD-L1 expression could be potentially ascribed to a down-regulation of AKT activity. We are currently investigating these hypotheses.
Jurkat cell line could serve dual actors for experimental models, either as leukemic tumor cells or immune effector $\mathrm{CD} 4+\mathrm{T}$ lymphocytes. Preliminary investigations showed that treatment of Jurkat cells with $40 \mu \mathrm{M}$ orlistat also induced a robust reduction of PD-1 expression. Binding of PD-1 to its ligand PD-L1 on tumor cells leads to the attenuation of T-cell proliferation, cytokine production, and cytolytic activities. ${ }^{61}$ Our data suggest that orlistat-mediated reduction of PD-L1 and PD-1 levels may lead to an overall decrease of the engagement of PD-1/PD-L1 axis in the tumor microenvironment and therefore to a vigorous increase of T-cell antitumor functionality. Interestingly, suppression of PD-L1 by ruxolitinib, a JAK1/JAK2 inhibitor, has been recently associated with a significant increase of the anti-tumor effects of anti-CTLA4 antibody therapy when administered after the immune checkpoint blockade therapy. ${ }^{62}$ Accordingly, the inhibition of PD-L1 expression mediated by FASN-inhibitor orlistat could potentially contribute to increase the response to CTLA4 blockade.

Moreover, our data show that orlistat does not interfere with the activity of a classical antileukemic agent such as MTX. Actually, the combined treatment with orlistat and MTX produces a slightly synergistic effect on T-cell leukemia cell growth.

Although further investigations are required, our observations uncover so far unexplored aspect underlying the orlistat-mediated mechanisms of tumor growth inhibition and support its employment in combination therapies against malignancies of hematological origin.

\section{Disclosure statement}

The authors declare that they have no competing interests.

\section{Funding}

This work was partially supported by the Funding of basic research activities 2017 from the Ministry of Education, University and Research (MIUR) to OF. SC is recipient of a fellowship from AIRC (Investigator Grant Project 17585).

\section{Author contributions}

GC, AA, OF, MPF and EB conceived and designed the study and wrote the paper. GC, MZ, $\mathrm{CV}, \mathrm{OF}, \mathrm{MPF}, \mathrm{MN}$ and MGC performed the experiments and analyzed the data, SC analyzed the data and performed statistical analysis. All 
authors reviewed the manuscript. In addition, all authors have read and approved the manuscript.

\section{Ethics approval and consent to participate}

Not applicable.

\section{Consent for publication}

Not applicable.

\section{Notes on contributors}

Giorgia Cioccoloni PhD. Research associate at the Department of Systems Medicine, University of Rome Tor Vergata, Rome, Italy. Expertise: clinical trials, in vivo and in vitro studies in cancer/obesity/nutrition.

Angelo Aquino. Associate Professor of Pharmacology, School of Medicine, Department of Systems Medicine, University of Rome Tor Vergata, Rome, Italy. Main area of interest: immunopharmacology of cancer.

Maria Notarnicola ScD. Board certified in Biochemistry, University of Bari, Italy. Main research activity: lipidome in metabolic diseases and cancer.

Maria Gabriella Caruso MD. Board certified in Internal Medicine and Biochemistry, Head of the Ambulatory of Clinical Nutrition of the De Bellis Research Hospital, Castellana Grotte, Bari, Italy. Research activity interest: lipid metabolism in cancer.

Enzo Bonmassar MD, PhD. Professor of Pharmacology and Research associate at the Consiglio Nazionale delle Ricerche (CNR, Italy). Main area of interest, role of Immunopharmacology in cancer treatment.

Manuela Zonfrillo. Graduate technician, National Research Council (Cnr, Rome Italy). Expertise: Cytometry, In vitro cell cultures.

Simona Caporali. Senior research assistant at the Istituto Dermopatico dell'Immacolata-IRCC. Expertise: molecular mechanisms involved in the pharmacological activity of anticancer drugs.

Cristina Villivà. Scholarship holder, biologist specialized in nutrition sciences.

Maria Pia Fuggetta. Senior researcher, National Research Council (Cnr, Rome Italy). Expertise: in vitro and in vivo studies in tumor and immunopharmacological field.

Ornella Franzese PhD. Assistant Professor of Pharmacology, School of Medicine, Department of Systems Medicine, University of Rome Tor Vergata, Rome, Italy. Main area of interest:
Immunotherapy of cancer, immunosenescence and mechanism of T-cell exhaustion.

\section{ORCID}

Manuela Zonfrillo (D) http://orcid.org/0000-00016457-2380

\section{References}

1 Ritchie MK, Johnson LC, Clodfelter JE, Pemble CW, Fulp $\mathrm{BE}$, Furdui CM, et al. Crystal structure and substrate specificity of human thioesterase 2: insights into the molecular basis for the modulation of fatty acid synthase. J Biol Chem. 2016;291: 3520-3530.

2 Wakil SJ. Fatty acid synthase, a proficient multifunctional enzyme. Biochemistry. 1989;28:4523-4530.

3 Hopperton KE, Duncan RE, Bazinet RP, Archer MC. Fatty acid synthase plays a role in cancer metabolism beyond providing fatty acids for phospholipid synthesis or sustaining elevations in glycolytic activity. Exp Cell Res. 2014;320:302-310.

4 Bauerschlag DO, Maass N, Leonhardt P, Verburg FA, Pecks U, Zeppernick F, et al. Fatty acid synthase overexpression: target for therapy and reversal of chemoresistance in ovarian cancer. J Transl Med. 2015;13:146.

5 Buckley D, Duke G, Heuer TS, O'Farrell M, Wagman AS, McCulloch W, et al. Fatty acid synthase - Modern tumor cell biology insights into a classical oncology target. Pharmacol Ther. 2017;177:23-31.

6 Bhatt AP, Jacobs SR, Freemerman AJ, Makowski L, Rathmell JC, Dittmer DP, et al. Dysregulation of fatty acid synthesis and glycolysis in non-Hodgkin lymphoma. PNAS. 2012;109: 11818-11823.

7 Cai Y, Wang J, Zhang L, Wu D, Yu D, Tian X, et al. Expressions of fatty acid synthase and HER2 are correlated with poor prognosis of ovarian cancer. Med Oncol. 2015;32: 391.

8 Mashima T, Seimiya H, Tsuruo T. De novo fatty-acid synthesis and related pathways as molecular targets for cancer therapy. Br J Cancer. 2009;100:1369-1372.

9 Zhao Y, Butler EB, Tan M. Targeting cellular metabolism to improve cancer therapeutics. Cell Death Dis. 2013;4:e532.

10 Wang H, Xi Q, Wu G. Fatty acid synthase regulates invasion and metastasis of colorectal cancer via Wnt signaling pathway. Cancer Med. 2016;5:1599-1606.

11 Liu ZL, Zhou Y, Luo QF, Hu M, Wang G, Huang S, et al. Inhibition of fatty acid synthase suppresses osteosarcoma cell invasion and migration. Indian J Pathol Microbiol. 2012;55: $163-169$.

12 Grube S, Dünisch P, Freitag D, Klausnitzer M, Sakr Y, Walter $\mathrm{J}$, et al. Overexpression of fatty acid synthase in human gliomas correlates with the WHO tumor grade and inhibition with Orlistat reduces cell viability and triggers apoptosis. J Neurooncol. 2014;118:277-287.

13 Martel PM, Bingham CM, McGraw CJ, Baker CL, Morganelli PM, Meng ML, et al. S14 protein in breast cancer cells: direct evidence of regulation by SREBP-1c, superinduction with progestin, and effects on cell growth. Exp Cell Res. 2006;312: 278-288.

14 Schcolnik-Cabrera A, Chávez-Blanco A, Domínguez-Gómez G, Taja-Chayeb L, Morales-Barcenas R, Trejo-Becerril C, et al. Orlistat as a FASN inhibitor and multitargeted agent for cancer therapy. Expert Opin Investig Drugs. 2018;27:475-489.

15 Sankaranarayanapillai M, Zhang N, Baggerly KA, Gelovani JG. Metabolic shifts induced by fatty acid synthase inhibitor orlistat in non-small cell lung carcinoma cells provide novel pharmacodynamic biomarkers for positron emission tomography and magnetic resonance spectroscopy. Mol Imaging Biol. 2013;15:136-147.

16 Agostini M, Almeida LY, Bastos DC, Ortega RM, Moreira FS, Seguin F, et al. The fatty acid synthase inhibitor orlistat reduces the growth and metastasis of orthotopic tongue oral squamous cell carcinomas. Mol Cancer Ther. 2014;13:585-595.

17 Huang HQ, Tang J, Zhou ST, Yi T, Peng HL, Shen GB, et al. Orlistat, a novel potent antitumor agent for ovarian cancer: proteomic analysis of ovarian cancer cells treated with Orlistat. Int J Oncol. 2012; 41:523-532. 
18 Seguin F, Carvalho MA, Bastos DC, Agostini M, Zecchin KG, Alvarez-Flores MP, et al. The fatty acid synthase inhibitor orlistat reduces experimental metastases and angiogenesis in B16F10 melanomas. Br J Cancer. 2012;107:977-987.

19 Cioccoloni G, Bonmassar L, Pagani E, Caporali S, Fuggetta MP, Bonmassar E, et al. Influence of fatty acid synthase inhibitor orlistat on the DNA repair enzyme O6-methylguanineDNA methyltransferase in human normal or malignant cells in vitro. Int J Oncol. 2015;47:764-772.

20 Pemble CWT, Johnson LC, Kridel SJ, Lowther WT. Crystal structure of the thioesterase domain of human fatty acid synthase inhibited by orlistat. Nat Struct Mol Biol. 2007;14: 704-709.

21 Knowles LM, Yang C, Osterman A, Smith JW. Inhibition of fatty-acid synthase induces caspase-8-mediated tumor cell apoptosis by up-regulating DDIT4. J Biol Chem. 2008;283: 31378-31384

22 Knowles LM, Axelrod F, Browne CD, Smith JW. A fatty acid synthase blockade induces tumor cell-cycle arrest by down-regulating Skp2. J Biol Chem. 2004;279:30540-30545.

23 Shen M, Tsai Y, Zhu R, Keng PC, Chen Y, Chen Y, et al. FASN-TGF- $\beta 1$-PD-L1 axis contributes to the development of resistance to NK cell cytotoxicity of cisplatin-resistant lung cancer cells. Biochim Biophys Acta Mol Cell Biol Lipids. 2018; 1863:313-322.

24 Liu H, Liu Y, Zhang JT. A new mechanism of drug resistance in breast cancer cells: fatty acid synthase overexpression-mediated palmitate overproduction. Mol. Cancer Ther. 2008;7: 263-270.

25 Sun C, Mezzadra R, Schumacher TN. Regulation and function of the PD-L1 checkpoint. Immunity. 2018;48:434-452.

26 Alsaab HO, Sau S, Alzhrani R, Tatiparti K, Bhise K, Kashaw SK, et al. PD-1 and PD-L1 checkpoint signaling inhibition for cancer immunotherapy: mechanism, combinations, and clinical outcome. Front Pharmacol. 2017;8:561.

27 Franzese O, Torino F, Fuggetta MP, Aquino A, Roselli M, Bonmassar E, et al. Tumor immunotherapy. drug-induced neoantigens (xenogenization) and immune checkpoint inhibitors. Oncotarget. 2017:8:41641-41669.

28 Goods BA, Hernandez AL, Lowther DE, Lucca LE, Lerner BA, Gunel M, et al. Functional differences between PD-1+ and PD-1- CD4+ effector $\mathrm{T}$ cells in healthy donors and patients with glioblastoma multiforme. PLoS ONE. 2017;12:e0181538.

29 Gong J, Chehrazi-Raffle A, Reddi S, Salgia R. Development of PD-1 and PD-L1 inhibitors as a form of cancer immunotherapy: a comprehensive review of registration trials and future considerations. J Immunother Cancer. 2018;6:8.

30 Renner K, Singer K, Koehl GE, Geissler EK, Peter K, Siska $\mathrm{PJ}$, et al. Metabolic hallmarks of tumor and immune cells in the tumor microenvironment. Front Immunol. 2017;8:248.

31 Kouidhi S, Ayed BF, Benammar Elgaaied A. Targeting Tumor Metabolism: A New Challenge to Improve Immunotherapy. Front Immunol. 2018; 9:353.

32 Pallasch CP, Schwamb J, Königs S, Schulz A, Debey S, Kofler $\mathrm{D}$, et al. Targeting lipid metabolism by the lipoprotein lipase inhibitor orlistat results in apoptosis of B-cell chronic lymphocytic leukemia cells. Leukemia. 2008;22:585-592.

33 Norberg M, Lindhagen E, Kanduri M, Rickardson L, Sundström C, Stamatopoulos K, et al. Screening for cytotoxic compounds in poor-prognostic chronic lymphocytic leukemia. Anticancer Res. 2012;32:3125-3136.

34 Goldberg JM, Silverman LB, Levy DE, Dalton VK, Gelber RD, Lehmann L, et al. Childhood T-cell acute lymphoblastic leukemia: the Dana-Farber Cancer Institute acute lymphoblastic leukemia consortium experience. JCO. 2003;21: 3616-3622.

35 Oudot C, Auclerc MF, Levy V, Porcher R, Piguet C, Perel Y, et al. Prognostic factors for leukemic induction failure in children with acute lymphoblastic leukemia and outcome after salvage therapy: the FRALLE 93 study. JCO. 2008;26:1496-1503.

36 Cervantes-Madrid D, Dueñas-González A. Antitumor effects of a drug combination targeting glycolysis, glutaminolysis and de novo synthesis of fatty acids. Oncol Rep. 2015;34: 1533-1542.

37 Sadowski MC, Pouwer RH, Gunter JH, Lubik AA, Quinn RJ, Nelson CC. The fatty acid synthase inhibitor triclosan: repurposing an anti-microbial agent for targeting prostate cancer. Oncotarget. 2014;5:9362-9381.
38 Chou TC, Talalay P. Quantitative analysis of dose-effect relationships: the combined effects of multiple drugs or enzyme inhibitors. Adv Enzyme Regul. 1984;22:27-55.

39 Bonmassar L, Fossile E, Scoppola A, Graziani G, Prete SP, Formica V, et al. Detection of circulating tumor cells is improved by drug-induced antigen up-regulation: preclinical and clinical studies. Anticancer Res. 2010;30:4721-4730.

40 Heuer TS, Ventura R, Mordec K, Lai J, Fridlib M, Buckley D, et al. FASN inhibition and taxane treatment combine to enhance anti-tumor efficacy in diverse xenograft tumor models through disruption of tubulin palmitoylation and microtubule organization and FASN inhibition-mediated effects on oncogenic signaling and gene expression. EBioMedicine. 2017; 16: $51-62$.

41 Sever R, Brugge JS. Signal transduction in cancer. Cold Spring Harbor Perspect Med. 2015; 5:a006098.

42 Corominas-Faja $\mathrm{B}$, Vellon $\mathrm{L}$, Cuyàs $\mathrm{E}$, Buxó $\mathrm{M}$, MartinCastillo B, Serra D, et al. Clinical and therapeutic relevance of the metabolic oncogene fatty acid synthase in HER2+ breast cancer. Histol Histopathol. 2017;32:687-698.

43 Jones SF, Infante JR. Molecular pathways: fatty acid synthase. Clin Cancer Res. 2015; 21:5434-5438.

44 Zhi J, Melia AT, Funk C, Viger-Chougnet A, Hopfgartner G, Lausecker B, et al. Metabolic profiles of minimally absorbed orlistat in obese/overweight volunteers. J Clin Pharmacol. 1996; 36:1006-1011.

45 Paulmurugan R, Bhethanabotla R, Mishra K, Devulapally R, Foygel K, Sekar TV, et al. Folate receptor-targeted polymeric micellar nanocarriers for delivery of orlistat as a repurposed drug against triple-negative breast cancer. Mol Cancer Ther. 2016;15:221-231.

46 Czumaj A, Zabielska J, Pakiet A, Mika A, Rostkowska O, Makarewicz W, et al. Vivo effectiveness of orlistat in the suppression of human colorectal cancer cell proliferation. Anticancer Res. 2019; 39:3815-3822.

47 Little JL, Wheeler FB, Koumenis C, Kridel SJ. Disruption of crosstalk between the fatty acid synthesis and proteasome pathways enhances unfolded protein response signaling and cell death. Mol Cancer Ther. 2008;7:3816-3824.

48 Peng J, Hamanishi J, Matsumura N, Abiko K, Murat K, Baba $\mathrm{T}$, et al. Chemotherapy induces programmed cell death-ligand 1 overexpression via the nuclear factor- $\kappa \mathrm{B}$ to foster an immunosuppressive tumor microenvironment in ovarian cancer. Cancer Res. 2015;75:5034-5045.

49 Van Der Kraak L, Goel G, Ramanan K, Kaltenmeier C, Zhang L, Normolle DP, et al. 5-Fluorouracil upregulates cell surface B7-H1 (PD-L1) expression in gastrointestinal cancers. J Immunother Cancer. 2016;4:65.

50 Ock CY, Kim S, Keam B, Kim S, Ahn YO, Chung EJ, et al. Changes in programmed death-ligand 1 expression during cisplatin treatment in patients with head and neck squamous cell carcinoma. Oncotarget. 2017;8:97920-97927.

51 Korde LA, Lusa L, McShane L, Lebowitz PF, Lukes L, Camphausen K, et al. Gene expression pathway analysis to predict response to neoadjuvant docetaxel and capecitabine for breast cancer. Breast Cancer Res Treat. 2010;119: 685-699.

52 Dong L, Lv H, Li W, Song Z, Li L, Zhou S, et al. Co-expression of PD-L1 and p-AKT is associated with poor prognosis in diffuse large B-cell lymphoma via PD-1/PD-L1 axis activating intracellular AKT/mTOR pathway in tumor cells. Oncotarget. 2016;7:33350-33362.

53 Muenst S, Schaerli AR, Gao F, Däster S, Trella E, Droeser RA, et al. Expression of programmed death ligand 1 (PD-L1) is associated with poor prognosis in human breast cancer. Breast Cancer Res Treat. 2014;146:15-24.

54 Kollmann D, Ignatova D, Jedamzik J, Chang YT, Jomrich G, Baierl A, et al. PD-L1 expression is an independent predictor of favorable outcome in patients with localized esophageal adenocarcinoma. Oncoimmunology. 2018;7:e1435226.

55 Knaus HA, Kanakry CG, Luznik L, Gojo I Immunomodulatory drugs: immune checkpoint agents in acute leukemia. CDT. 2017;18:315-331.

56 Dong H, Strome SE, Salomao DR, Tamura H, Hirano F, Flies DB, et al. Tumor-associated B7-H1 promotes T-cell apoptosis: a potential mechanism of immune evasion. Nat Med. 2002;8: 793-800.

57 Clark CA, Gupta H, Sareddy GR, Pandeswara S, Lao S, Yuan $\mathrm{B}$, et al. Tumor-intrinsic PD-L1 signals regulate cell growth, 
pathogenesis and autophagy in ovarian cancer and melanoma. Cancer Res. 2016;76:6964-6974.

58 Yan F, Pang J, Peng Y, Molina JR, Yang P, Liu S. Elevated cellular PD1/PD-L1 expression confers acquired resistance to cisplatin in small cell lung cancer cells. PLoS One. 2016;11: e0162925.

59 Lastwika KJ, Wilson W, Li QK, Norris J, Xu H, Ghazarian $\mathrm{SR}$, et al. Control of PD-L1 expression by oncogenic activation of the AKT-mTOR Pathway in non-small cell lung cancer. Cancer Res. 2016;76:227-238.
60 Li L, Pilo GM, Li X, Cigliano A, Latte G, Che L, et al. Inactivation of fatty acid synthase impairs hepatocarcinogenesis driven by AKT in mice and humans. J Hepatol. 2016;64:333-341.

61 Keir ME, Butte MJ, Freeman GJ, Sharpe AH. PD-1 and its ligands in tolerance and immunity. Annu Rev Immunol. 2008; 26:677-704.

62 Pitroda SP, Stack ME, Liu GF, Song SS, Chen L, Liang H, et al. JAK2 inhibitor SAR302503 abrogates PD-L1 expression and targets therapy-resistant non-small cell lung cancers. Mol Cancer Ther. 2018;17:732-739. 